\title{
ÍNDICE DE LEVANTAMENTO DA EQUAÇÃO DO NIOSH E LOMBALGIA ${ }^{1}$
}

\section{LIFTING INDEX OF THE NIOSH LIFTING EQUATION AND LOW BACK PAIN}

\author{
Eliana Remor Teixeira* E-mail: elianart2002@yahoo.com.br \\ Maria Lúcia Ribeiro Okimoto**E-mail: Iucia.demec@ufpr.br \\ Leila Amaral Gontijo* E-mail: leila@deps.ufsc.br \\ *Universidade Federal de Santa Catarina, Florianópolis, SC \\ ${ }^{*}$ Universidade Federal do Paraná, Curitiba, PR
}

\begin{abstract}
Resumo: Este estudo tem por objetivo avaliar a relação entre o Índice de Levantamento obtido mediante a aplicação da Equação de Levantamento do NIOSH e a incidência de lombalgia entre quarenta e oito trabalhadores envolvidos nas tarefas de levantamento manual de cargas. Aplicou-se a equação em onze tarefas e realizou-se entrevista com os trabalhadores. As condições mais desfavoráveis apresentavam-se no destino do levantamento. As variáveis da tarefa que mais contribuíram para os valores inadequados do Índice de Levantamento foram: a distância horizontal, a frequência de levantamentos e a distância vertical; além do peso elevado da carga. A incidência de lombalgia nos últimos doze meses foi de $19 \%$, enquanto que a incidência da lombalgia relacionada ao trabalho, no mesmo período, foi de $10 \%$. Em $72,7 \%$ das tarefas avaliadas o Índice de Levantamento Composto foi superior a três, sendo consideradas como de alto risco ergonômico.
\end{abstract}

Palavras-chave: Equação de levantamento do $\mathrm{NIOSH}$. Índice de levantamento. Levantamento manual de cargas. Lombalgia. Ergonomia.

Abstract: The purpose of this study is to assess the relationship of the Lifting Index obtained through the application of the NIOSH Lifting Equation and the incidence of low back pain among forty-eight workers involved in manual lifting tasks. It was applied the equation in eleven tasks and the workers were interviewed. The most unfavorable conditions presented themselves in the lifting destination. The variables that most contributed to the inadequate values of the Lifting Index were: the horizontal location, the lifting frequency and the vertical distance, beyond the high weight of the load. The incidence of low back pain in the last twelve months was 19\%, whereas the incidence of work-related low back pain in the same period was $10 \%$. In $72.7 \%$ of the tasks evaluated the Composite Lifting Index was more than three, which are considered as high ergonomic risk.

Keywords: NIOSH lifting equation. Lifting Index. Low back pain. Manual lifting. Ergonomics.

\section{INTRODUÇÃO}

Os distúrbios da coluna lombar são frequentes e causam transtornos para o trabalhador, a empresa e a sociedade em geral. É relatado na bibliografia que 70 a

\footnotetext{
${ }^{1} \mathrm{O}$ texto é inédito. $\mathrm{O}$ estudo não foi financiado, embora a primeira autora tenha sido bolsista da CAPES. É resultado de dissertação de mestrado e não há conflito de interesses.
}

Revista Produção Online. Florianópolis, SC, v.11, n. 3, p. 735-756, jul./set., 2011. 
$80 \%$ da população apresentam algum episódio de lombalgia durante a vida (MARRAS, 2000, QUINN, 2002).

Entre as causas de lombalgia apontam-se diversos fatores de risco ocupacionais, entre os quais se evidenciam como fator causal as atividades de levantamento manual de cargas. Assim, a lombalgia representa um importante problema de saúde ocupacional nos países industrializados, contribuindo com 20 a $30 \%$ de todos os afastamentos do trabalho por doença e representando $50 \%$ do total de custos diretos a eles relacionados (KERR et al., 2001).

Com o propósito de avaliar a demanda física das tarefas de levantamento manual de cargas e estimar o risco de lesões por sobrecarga e lombalgia, relacionado a cada tarefa específica (GARG, 1995), foi concebida a Equação de Levantamento Revisada do National Institute for Occupational Safety and Health $(E L N)$.

A aplicação da ELN fornece o Índice de Levantamento (IL), que representa uma estimativa do estresse físico associado à tarefa avaliada. Segundo Waters e colaboradores (1993) existe relação entre o IL e o risco de lombalgia. Por sua vez, Garg (1995) afirma que quando o IL for abaixo de um, a tarefa é considerada segura. IL acima de um significa que pode apresentar risco para alguns trabalhadores e à medida que o IL aumenta, aumenta o risco da tarefa. Salienta, ainda, que a relação entre IL e risco de lesão por sobrecarga não é linear. Dessa forma, um trabalhador com um IL de 3 não está duas vezes mais exposto ao risco que um trabalhador cuja tarefa possuiu um IL de 1,5.

No estudo epidemiológico de Waters e colaboradores (1999a) sobre incidência de lombalgia na atividade de levantamento de cargas e a aplicação da equação, as tarefas foram classificadas de acordo com os valores de IL em quatro grupos: $\mathrm{IL}=0 ; 0<\mathrm{IL} \leq 1 ; 1<\mathrm{IL} \leq 2 ; 2<\mathrm{IL} \leq 3$; e $\mathrm{IL}>3$. O referido estudo contou com 204 trabalhadores envolvidos em 50 tarefas de levantamento de cargas em quatro indústrias. Cinquenta e nove por cento das tarefas avaliadas eram tarefas simples; no grupo $2<\mathrm{IL} \leq 3(\mathrm{n}=121)$, houve correlação estatística entre IL e lombalgia. Os dados foram insuficientes para concluir sobre o risco nos demais grupos. Os autores recomendaram futuros estudos com um tamanho de amostra maior para possibilitar avaliar a existência ou não de correlação nos demais grupos. 
Visando compreender o comportamento da equação no ambiente de trabalho, discutir as dificuldades na sua aplicação e identificar a ocorrência de lombalgia entre os trabalhadores envolvidos em atividades de levantamento manual de cargas, foi proposto o presente estudo que objetiva:

- avaliar a relação entre o IL e a incidência de lombalgia nos últimos doze meses em quarenta e oito trabalhadores envolvidos nas tarefas de Levantamento Manual de Cargas, na Região Metropolitana de Curitiba - PR;

- descrever os fatores mais desfavoráveis das tarefas;

- evidenciar a utilidade da ELN para avaliação e planejamento de melhorias das tarefas de levantamento manual de cargas;

- discutir os aspectos epidemiológicos da lombalgia relacionada ao levantamento manual de cargas.

\section{FUNDAMENTAÇÃO TEÓRICA}

\subsection{Lombalgia}

A lombalgia pode ser definida como dor, tensão muscular, rigidez localizada abaixo da margem costal e acima da dobra glútea inferior, com ou sem dor nas pernas. É um distúrbio que afeta muitas pessoas durante a vida e está associada a elevados custos diretos e indiretos. Os custos estão associados à utilização dos serviços de saúde, absenteísmo no trabalho e incapacidade dos quais resultam consequências importantes para o trabalhador, seus familiares, empregadores e para a sociedade em geral (TULDER; KOES, BOMBARDIER, 2002).

A lombalgia é um sintoma e não uma doença; não é simplesmente aguda ou crônica, mas pode flutuar ao longo do tempo com recorrências ou exacerbações. Devido a essas características que se modificam ao longo do tempo, é que existem divergências nos resultados apresentados por diversos estudos em relação à incidência e/ou prevalência da lombalgia. Dependendo da fase do fenômeno em que foram obtidos os dados, os resultados encontrados podem ser diferentes. Assim, alguns pesquisadores podem investigar as queixas dos trabalhadores mediante questionários e outros apenas trabalhar com dados coletados dos diagnósticos 
registrados no Serviço Médico das empresas ou Prestadoras de Serviço de Saúde, ou de outro banco de dados (TULDER; KOES, BOMBARDIER, 2002).A progressão da lombalgia ao longo do tempo pode ser compreendida mediante a ilustração na figura 1.

Figura 1- Progressão da lombalgia ao longo do tempo

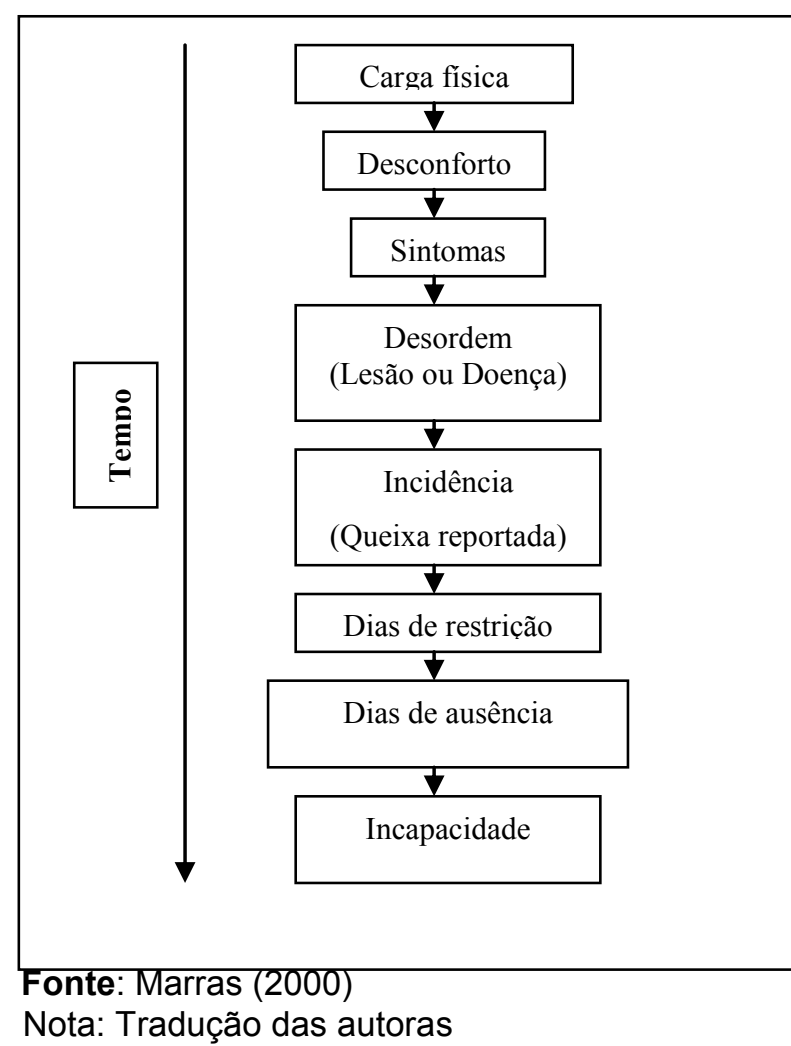

É unanimidade entre os pesquisadores acreditarem que, aproximadamente, $90 \%$ de todos os sujeitos com lombalgia apresentarão lombalgia não específica, ou seja, de causa desconhecida (BYRNS et al., 2002, TULDER; KOES; BOMBARDIER; 2002). Os sintomas mais importantes da lombalgia não específica são dor e incapacidade. De acordo com os referidos autores, a lombalgia não específica pode ser classifica em relação à duração da queixa em:

a) aguda: quando os sintomas persistem por menos de seis semanas;

b) subaguda: quando os sintomas permanecem por um período entre seis semanas e três meses;

c) crônica: quando os sintomas persistem por mais de três meses. 
Byrns e colaboradores (2002) salientam a importância da intervenção precoce na prevenção e no tratamento da lombalgia já que, quanto maior for o período de duração dos sintomas e do afastamento do trabalho, menor será a chance de o trabalhador retornar com as mesmas condições físicas existentes antes do afastamento. Logo, o tratamento precoce evitará que episódios de lombalgia aguda evoluam para casos crônicos. A fase subaguda, entre oito e doze semanas, é também uma fase muito importante na prevenção da lombalgia crônica.

As causas da lombalgia crônica são desconhecidas e podem estar associadas à osteoartrose, à degeneração do disco intervertebral, à osteoporose e a outras. Segundo Marras (2000), em 70\% dos pacientes com lombalgia crônica não é possível o estabelecimento de diagnóstico específico.

É estimado que apenas $15 \%$ dos casos de lombalgia são precedidos de causa orgânica subjacentes ou lesão específica. A maioria dos casos de lombalgia deve-se à sobrecarga dos elementos músculos-esqueléticos, que causa inflamação e produz dor. A exposição repetida a cargas físicas resulta em dose cumulativa. Quando a dose ultrapassa a capacidade de tolerância tecidual do indivíduo, surge a dor. Byrns e colaboradores (2002), conforme ilustração da figura 2, apresentam esquematicamente explicação sobre a etiologia da lombalgia ${ }^{2}$.

Figura 2 - Explicação da etiologia da lombalgia mediante da exposição à sobrecarga física

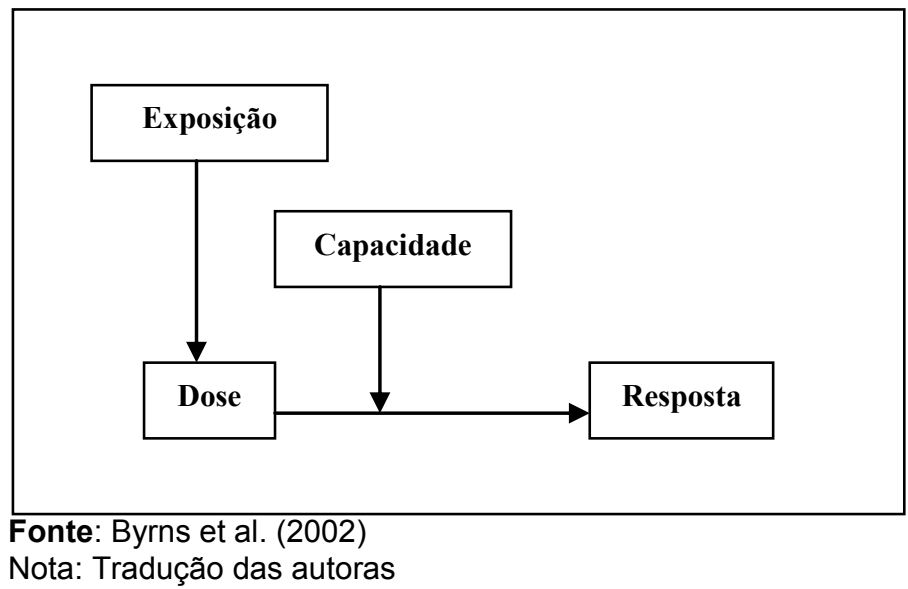

\footnotetext{
${ }^{2}$ Para aprofundar o estudo sobre os fatores contributivos da lombalgia relacionada ao trabalho, ver Byrns e colaboradores (2002).
} 
A avaliação da sobrecarga para a coluna lombar é complexa, visto a multiplicidade de estruturas envolvidas, tais como os músculos, os ligamentos e as partes moles dessa região. Vários estudos concluíram que a sobrecarga sobre a coluna varia em função dos movimentos repetitivos, flexão, movimentos de torção e flexão lateral do tronco (MARRAS, 2000).

Marras (2000) ensina que existem três formas de explicar como a sobrecarga pode ocasionar a lesão na região lombar, conforme se observa na figura 3, de acordo com a lógica biomecânica, variabilidade da carga e tolerância do trabalhador.

Figura 3 - Explicação biomecânica sobre o mecanismo de lesão lombar por sobrecarga (a) de acordo com a lógica biomecânica - quando a sobrecarga excede a tolerância tecidual, a lesão ocorre; (b) explicação biomecânica para o trauma cumulativo devido à variabilidade aumentada da carga, com algumas excedendo a tolerância; (c) explicação biomecânica para o trauma cumulativo devido à redução da tolerância.

(a

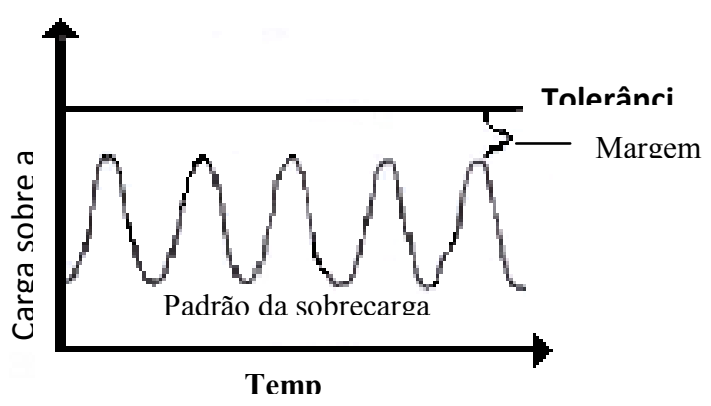

de

(b

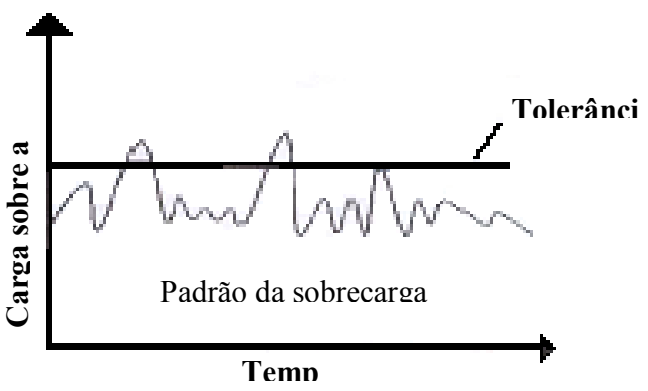

(c

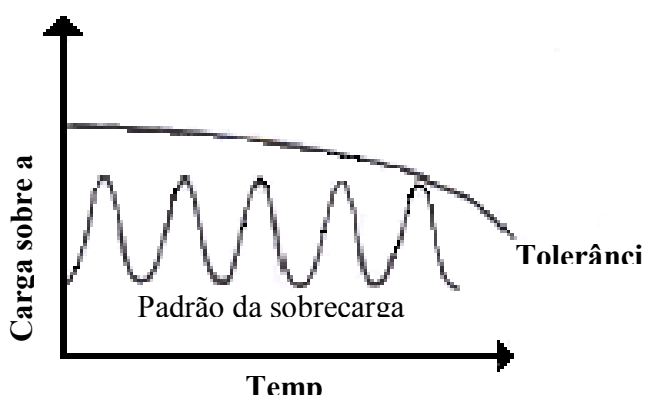

Fonte: Marras (2000)

Nota: Tradução das autoras

Revista Produção Online. Florianópolis, SC, v.11, n. 3, p. 735-756, jul./set., 2011. 
Hartvigsen e colaboradores (2001) encontraram correlação entre o trabalho fisicamente pesado e a lombalgia, num estudo prospectivo de cinco anos. Thorbjornsson e colaboradores (2000) também observaram tal correlação acrescida de trabalho sedentário e vibração de corpo inteiro, além do pouco controle sobre o trabalho, entre as mulheres, e problemas de relacionamento interpessoal no trabalho, entre os homens.

Couto (1995) atribuiu às atividades de levantamento de cargas as causas das lombalgias relacionadas ao trabalho decorrentes de protrusão do disco intervertebral.

Lin e colaboradores (2002) estudaram as características das tarefas de levantamento de cargas entre 191 pacientes de uma clínica de fisioterapia com queixas de lombalgia cujo trabalho era centrado nesse tipo de tarefa. Os fatores mais desfavoráveis identificados foram: peso da carga (em $63,35 \%$ o peso excedia a $30 \mathrm{~kg}$ ), tamanho da carga (em 74,87\%, o tamanho ultrapassava a dimensão entre os ombros do trabalhador), postura inadequada, como rotação do tronco durante o levantamento e flexão do tronco, sem flexão dos joelhos.

\subsection{Equação de Levantamento do NIOSH - ELN}

A ELN é baseada num modelo multiplicativo que fornece um peso para cada uma das seis variáveis da tarefa. Os pesos são expressos como coeficientes que servem para reduzir a constante de carga, que representa o peso máximo recomendado para ser levantado em condições ideais. O Limite de Peso Recomendado (LPR) é o produto da equação e é definido como o peso da carga que aproximadamente todos os trabalhadores saudáveis poderiam suportar por um período de até 8 horas diárias, sem aumentar o risco de desenvolverem lombalgia relacionada ao trabalho ( $\mathrm{NIOSH}, 1994)$.

O LPR é obtido através da seguinte equação:

$\mathrm{LPR}=\mathrm{Cc} \times \mathrm{FH} \times \mathrm{FV} \times \mathrm{FD} \times \mathrm{FA} \times \mathrm{FF} \times \mathrm{FP}$

ou

$\operatorname{LPR}=23 \times[25 / \mathrm{H}] \times[1-(0,003|\mathrm{~V}-75|)] \times[0,82+(4,5 / D)] \times[1-(0,0032 \mathrm{~A})] \times \mathrm{FF} \times \mathrm{FP}$

Revista Produção Online. Florianópolis, SC, v.11, n. 3, p. 735-756, jul./set., 2011. 
Uma vez calculado o LPR para uma dada tarefa de levantamento de cargas, ele é comparado com o peso real da carga levantada. Essa relação fornece o Índice de Levantamento (IL). Onde:

$\mathrm{IL}=\mathrm{PC} / \mathrm{LPR}$

$P C=$ Peso real da carga (em quilogramas)

LPR + Limite de Peso Recomendado (em quilogramas)

Cada um dos coeficientes é estabelecido a partir do valor de cada variável encontrada na tarefa específica: distância horizontal $(H)$, distância vertical $(V)$, distância vertical percorrida pela carga (D), ângulo de assimetria $(A)$, frequência de levantamento $(F)$ e pega $(P)$. A representação das variáveis podem ser observadas nas figuras 4 e 5 a seguir.

Cada fator pode ser calculado conforme a fórmula apresentada anteriormente ou obtido por meio das tabelas 1, 2, 3, 4, 5 e 7 do NIOSH (NIOSH, 1994). Para um estudo aprofundado sobre a aplicação da equação, ver NIOSH (1994) e Teixeira (2004). Detalhes sobre a concepção da expressão matemática da ELN podem ser encontrados em Waters e colaboradores (1993).

Figura 4 - Representação gráfica das variáveis H , V

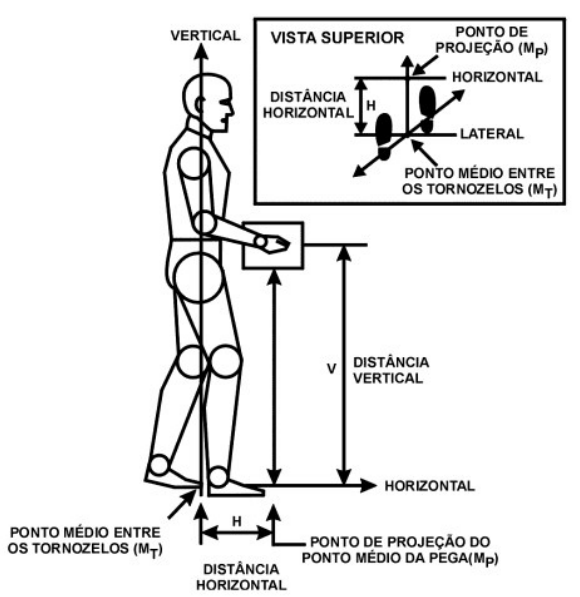

Fonte: Adaptado de NIOSH (1994)

Revista Produção Online. Florianópolis, SC, v.11, n. 3, p. 735-756, jul./set., 2011. 
Figura 5 - Representação gráfica do ângulo de assimetria (A)

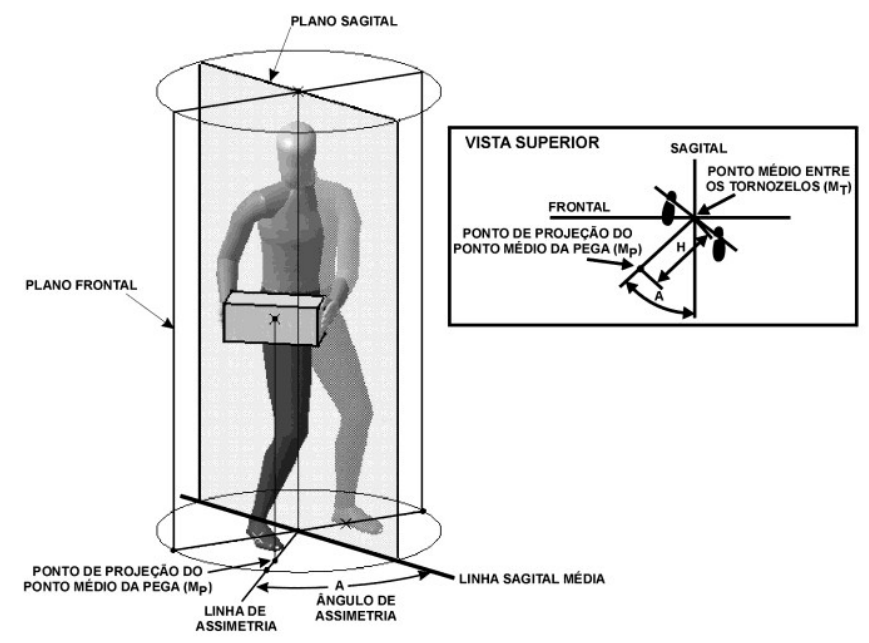

Fonte: Adaptado de NIOSH (1994)

A ELN é uma das ferramentas útil na avaliação das tarefas de levantamento de cargas e vem sendo utilizada em diversos países, inclusive no Brasil. Porém, nem todas as tarefas de levantamento poderão ser avaliadas mediante a referida equação.

A complexidade das tarefas existentes nas situações reais de trabalho dificulta a aplicação da equação. Podem surgir dificuldades na determinação do Fator Frequência decorrentes da grande variabilidade das mesmas, assim como na determinação da tarefa da possibilidade de avaliação de determinada tarefa segundo a ELN.

Dempsey (2002) e Waters, Putz-Anderson e Baron (1998), após estudos sobre a precisão nas medidas entre os usuários da equação que haviam recebido treinamento prévio sobre a sua aplicação, concluíram que a variabilidade estabelecia-se, normalmente, dentro de níveis aceitáveis. Porém, na opinião de Burdorf e Van Der Beek (1999), no estudo de Waters, Putz-Anderson e Baron (1998), por ter sido realizado em laboratório, os efeitos dos erros nas medições sobre o LPR foram bem menores do que pode ocorrer em situações reais de trabalho.

Outro aspecto levantado pelos autores acima citados foi o fato de a frequência e o peso terem sido estabelecidos de forma hipotética no estudo de 
Waters, Putz-Anderson e Baron (1998), principalmente porque a frequência é difícil de ser avaliada em campo e é a variável que apresenta o maior peso na determinação do LPR. Então, Waters (1999b) justifica o modelo do estudo considerando que, geralmente, os erros de medição podem ser de três tipos: $\left(1^{\circ}\right)$ erros atribuídos à variabilidade na execução da tarefa; $\left(2^{\circ}\right)$ erros resultantes da variabilidade da demanda da tarefa; e $\left(3^{\circ}\right)$ erros devido a dificuldades de medição das características de interesse da tarefa. $O$ autor refere ainda que, no estudo em destaque, havia interesse em limitar a pesquisa à medição de erro do terceiro tipo.

Devido à grande sensibilidade do Limite de Peso Recomendável (LPR) e do IL a erros na frequência e na distância horizontal, esses parâmetros devem ser priorizados, em relação à precisão das medidas. Como a precisão nas medidas é fundamental para a correta aplicação da equação, conforme abordado anteriormente, é recomendável que os usuários recebam treinamentos teórico e prático, visando maior padronização dos resultados (DEMPSEY et al., 2001). Teixeira (2004) propôs um procedimento sistematizado para a obtenção das variáveis da tarefa necessárias à aplicação da equação que contribui para a precisão das medições.

No estudo de Chung e Dohyung (2000) em uma indústria de manufatura da Coréia do Sul, que apresentava alta incidência de lombalgia, os índices de levantamento encontrados variaram de 0,86 a 8,8 (média de 2,73) e 3,7 a 18,9 (média de 11,12) em setores distintos. Foi observado que a maioria das tarefas excedia o LPR e as variáveis que mais contribuíram para a condição desfavorável das mesmas foram distância horizontal $(H)$ e ângulo de assimetria $(A)$. Todos os trabalhadores das tarefas em que o Índice de Levantamento Composto (ILC) excedeu a cinco referiram lombalgia. Para esclarecimentos sobre o ILC, ver NIOSH (1994).

No estudo preliminar a respeito da percepção dos trabalhadores sobre a exposição ao risco nas tarefas de levantamento, Yeung e coboradores (2002) observaram correlação entre o grau de risco percebido pelos trabalhadores para realizarem a tarefa e os valores de IL apresentados pelas mesmas. 


\section{METODOLOGIA}

A metodologia do presente estudo consistiu de revisão da literatura sobre lombalgia e sobre a ELN; obtenção de uma amostra de quarenta e oito trabalhadores em onze tarefas de levantamento manual de cargas em seis empresas da região metropolitana de Curitiba, Paraná, Brasil, do ramo metalmecânico e de alimentos; entrevista com 100\% dos trabalhadores envolvidos nas onze tarefas para obtenção dos dados epidemiológicos; aplicação da ELN de acordo com a metodologia proposta em NIOSH (1994) e procedimento proposto por Teixeira (2004) para obtenção das variáveis da equação e que pode ser observado em Okimoto e Teixeira (2009). Os dados foram registrados no Epi Info 2002, versão 2. ${ }^{3}$ Utilizou-se também o programa Statgraphics, versão 6.0, para complementar as análises estatísticas dos dados.

A participação dos trabalhadores foi voluntária. As tarefas foram selecionadas mediante observações de uma das pesquisadoras, acompanhada de um representante dos trabalhadores e de um do empregador, em cada empresa participante da pesquisa.

\section{RESULTADOS E DISCUSSÃO}

Cada uma das onze tarefas avaliadas requereu, em média, de quatro a oito horas em campo para medições e registro das variáveis. As tarefas de levantamento de cargas avaliadas foram as seguintes:

a) Tarefa 1 (T1) - Palletização de chapas de aço, com peso médio de $33 \mathrm{~kg}$;

b) Tarefa 2 (T2) - Acondicionamento de peças metálicas na embalagem, cuja carga apresenta peso médio de $5 \mathrm{~kg}$;

c) Tarefa 3 (T3) - Palletização de sacas de $25 \mathrm{~kg}$ de farináceo;

d) Tarefa 4 (T4) - Palletização de sacas de $10 \mathrm{~kg}$ de farináceo;

e) Tarefa 5 (T5) - Palletização de sacas de $25 \mathrm{~kg}$ de farináceo;

\footnotetext{
${ }^{3}$ Sistema de processamento de texto, banco de dados e estatística para Epidemiologia em Microcomputadores - programa de domínio público que pode ser livremente copiado através do site: www.cdc.gov/epiinfo.
} 
f) Tarefa 6 (T6) - Tarefa composta de 4 subtarefas de levantamento de caixas de $40 \mathrm{~kg}$, contendo peças metálicas;

g) Tarefa 7 (T7) - Palletização de caixas de 5,4 kg, contendo líquido;

h) Tarefa 8 (T8) - Levantamento de ganchos com peças metálicas, com peso médio de 13,80 kg e peso máximo de 36 kg;

i) Tarefa 9 (T9) - Levantamento de balde de $40 \mathrm{~kg}$, contendo peças metálicas;

j) Tarefa 10 (T10) - Operar máquina afiadora de peças metálicas, tendo que levantar cargas que variam de 19 a $40 \mathrm{~kg}$ (tarefa composta de cinco subtarefas de levantamento de carga).

k) Tarefa 11 (T11) - Palletização de caixas de 10 kg.

Os registros das avaliações das tarefas podem ser observados em Teixeira (2004).

\subsection{Dados pessoais}

Todos os trabalhadores eram do sexo masculino. Em relação à faixa etária, observou-se que $54,2 \%$ dos trabalhadores tinham idade entre vinte e vinte e nove anos e que $89,6 \%$ apresentavam idade inferior a quarenta anos.

Os trabalhadores apresentavam estatura média de $171,3 \mathrm{~cm}$, com desvio padrão de $5,51 \mathrm{~cm}$. Observou-se que a população da amostra tinha estatura elevada, pois apenas $14,7 \%$ apresentavam estatura inferior a $166 \mathrm{~cm}$.

Os valores do IMC entre os trabalhadores da amostra revelaram que $71 \%$ apresentaram peso dentro do normal, ou seja, IMC $<25$. Desses, somente três trabalhadores apresentaram IMC < que 20, com os seguintes valores: 17,24; 18,8 e 19,44. Somente 4\% dos trabalhadores da amostra apresentaram Obesidade Grau II e não foi registrado nenhum caso de Obesidade Grau III. Foi utilizada a classificação de obesidade de acordo com o IMC apresentado por Pi-Sunyer (1990). A baixa incidência de obesidade entre esses trabalhadores pode ser explicada pela grande demanda física dessas tarefas. 


\subsection{Dados ocupacionais}

Da população pesquisada, apenas $41,7 \%$ dos trabalhadores exerciam a função há mais de 12 meses.Vinte e cinco trabalhadores (52,1\%) tinham menos de um ano na função. Salienta-se, ainda, que vinte e três trabalhadores $(47,9 \%)$ exerciam tal função há menos de seis meses. Somente um trabalhador tinha mais de dez anos na função. Esses dados evidenciam o elevado turnover nas tarefas avaliadas. Observou-se que, em duas tarefas de empresas distintas, a mão-de-obra era terceirizada. Nesses casos, o turnover também ficou bastante evidente pelo pouco tempo na função.

Vinte e dois trabalhadores $(45,8 \%)$, durante a vida, trabalharam entre um a cinco anos em tarefas de levantamento de cargas. Apenas quatro $(8,3 \%)$ tinham menos de um ano, e $16(33,4 \%)$ trabalharam durante mais de dez anos nesse tipo de atividade.

\subsection{Dados relacionados à lombalgia}

A incidência de lombalgia durante a vida foi de 46\% (22 trabalhadores) na população estudada. A incidência de lombalgia nos últimos doze meses entre esses trabalhadores foi de $19 \%$, percentual que representa nove dos quarenta e oito trabalhadores da amostra. Dos nove (19\%) trabalhadores que apresentaram lombalgia nos últimos doze meses, cinco (10\% da amostra) relacionaram o sintoma com as condições da tarefa de levantamento.

A incidência de lombalgia por acidente de trabalho foi de $4 \%$, o que representa dois dos quarenta e oito trabalhadores da amostra. Esses dados foram obtidos mediante entrevistas com os trabalhadores, não tendo sido confirmado se houve registro mediante Comunicação de Acidente do Trabalho (CAT).

O número de afastamentos do trabalho nos últimos doze meses por lombalgia foi dois, o que representa $4 \%$ dos trabalhadores. Nota-se que o número de afastamentos por lombalgia nos últimos doze meses coincidiu com a incidência de acidente de trabalho por lombalgia. Os trabalhadores que se afastaram do trabalho por lombalgia foram, na verdade, aqueles que relataram terem sido acometidos por 
lombalgia devido a acidente de trabalho. Esses dados ilustram a importância da prevenção da lombalgia melhorias mediante condições das tarefas de levantamento, contribuindo, assim, para a redução do absenteísmo na empresa.

\subsection{Dados relacionados à aplicação da ELN}

Para análise dos dados relacionados à aplicação da ELN nas onze tarefas avaliadas, consideraram-se, para cada tarefa, os dados da subtarefa mais desfavorável. Dado o valor ideal para cada variável: $\mathrm{H} \leq 25 \mathrm{~cm} ; \mathrm{V}=75 \mathrm{~cm} ; A=0$; $D$ $=\leq 25 \mathrm{~cm} ; \mathrm{F} \leq 0,2$ levs $/ \mathrm{min}$, de acordo com as tabelas dos coeficientes apresentadas pelo NIOSH (1994), pode-se observar a condição de cada variável em cada tarefa.

Os valores de $\mathrm{H}$ na origem, em geral, apresentaram-se menores que no destino, conforme pode ser observado no gráfico 1, a seguir. Isso significa que, na origem do levantamento, a carga estava mais próxima do corpo do trabalhador.

Gráfico 1 - Valores da variável $H$, na origem $\left(H_{\circ}\right)$ e no destino $\left(H_{d}\right)$ do levantamento

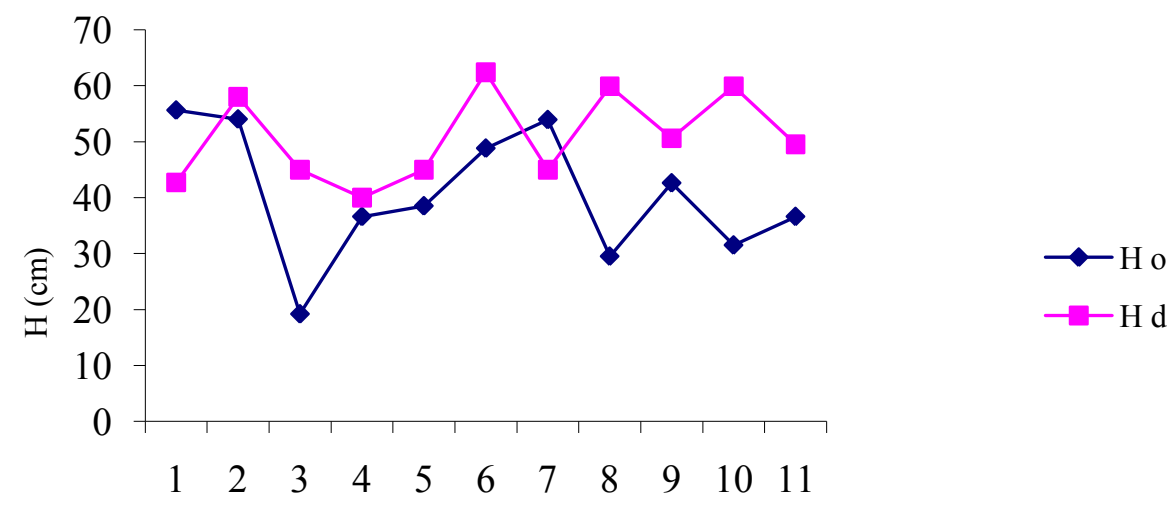

Tarefas

O valor do peso real da carga que estava sendo levantada, em geral, era superior ao Limite de Peso Recomendado (LPR), obtido mediante aplicação da equação. Somente a Tarefa 7 (T7) apresentou o peso real da carga inferior ao LPR, conforme se observa no gráfico 2. Constatam-se, assim, que as tarefas apresentam 
condições desfavoráveis aos trabalhadores, pois os mesmos estão levantando a carga de 2 a 8 vezes mais do que o recomendado conforme o LPR obtido.

Gráfico 2 - Valores do LPR e peso médio da carga

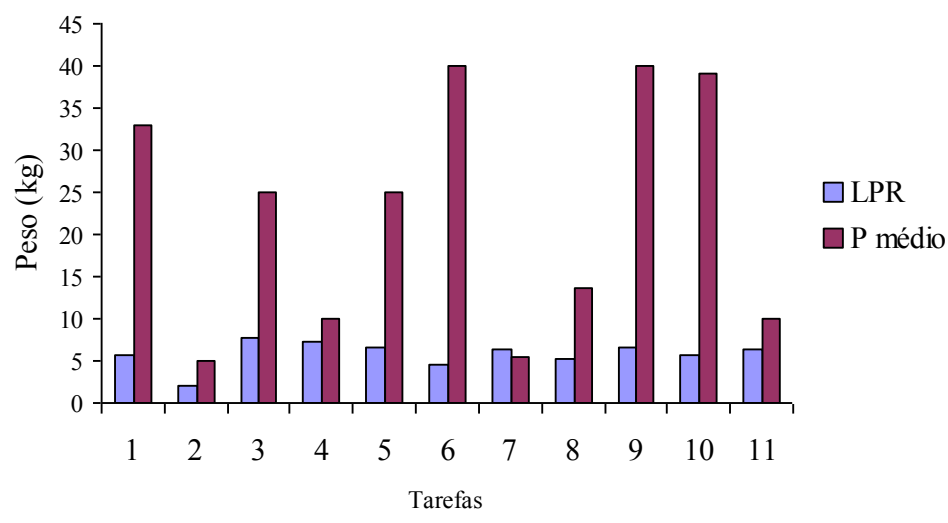

As tarefas que apresentaram o maior e o menor valor de ILC foram as Tarefas 6 (ILC = 8,84) e 7 (ILC = 1,5), respectivamente, conforme ilustração do gráfico 3. A Tarefa 6 (T6) era composta de 4 subtarefas de levantamento de caixas de $40 \mathrm{~kg}$, contendo peças metálicas e a Tarefa 7 (T7) era a Palletização de caixas de $5,4 \mathrm{~kg}$, contendo líquido.

Gráfico 3 - Valores do ILC das tarefas de levantamento manual de carga

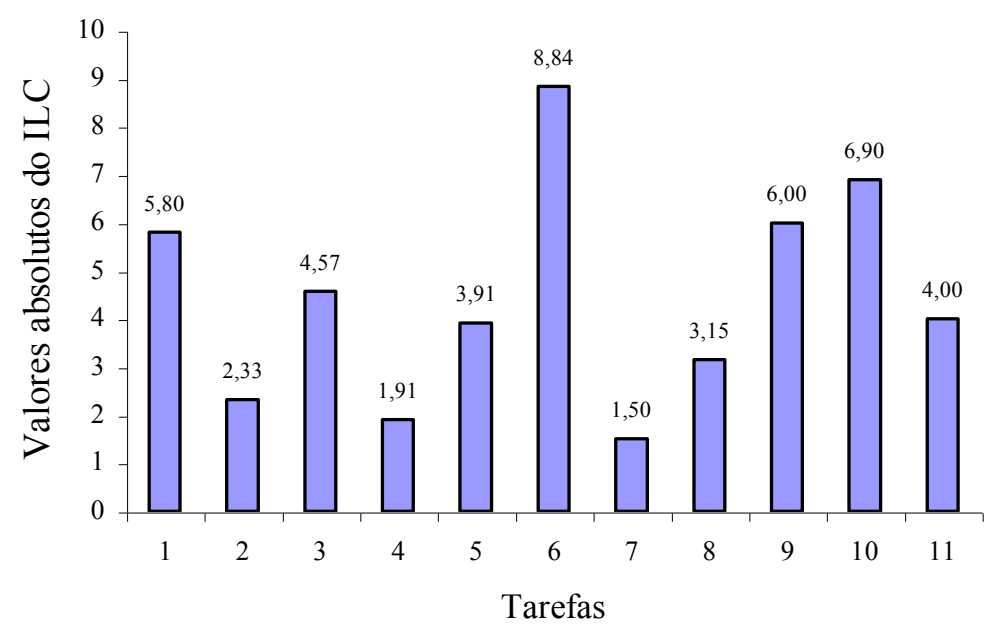

Revista Produção Online. Florianópolis, SC, v.11, n. 3, p. 735-756, jul./set., 2011. 
As tarefas avaliadas podem ser visualizadas segundo o valor do ILC, em ordem decrescente, conforme ilustrado na tabela 1. Dessa forma, pode-se comparálas.

Tabela 1 - Tarefas de levantamento de carga segundo ordem decrescente do ilc

\begin{tabular}{ccc}
\hline CLASSIFICAÇÃO & TAREFA & ILC \\
\hline 1 & T6 & 8,84 \\
2 & T10 & 6,9 \\
3 & T9 & 6,0 \\
4 & T1 & 5,8 \\
5 & T3 & 4,57 \\
6 & T11 & 4,0 \\
7 & T5 & 3,91 \\
8 & T8 & 3,15 \\
9 & T2 & 2,33 \\
10 & T4 & 1,91 \\
11 & T7 & 1,5 \\
\hline
\end{tabular}

Oito tarefas - T3, T4, T5, T6, T8, T9, T10, T11- apresentaram condição mais desfavorável no destino do levantamento, conforme se observa no gráfico 4 , pelos menores valores do LPR evidenciados no destino em relação aos valores na origem do levantamento.

Gráfico 4 - Valores do LPR na origem e destino do levantamento

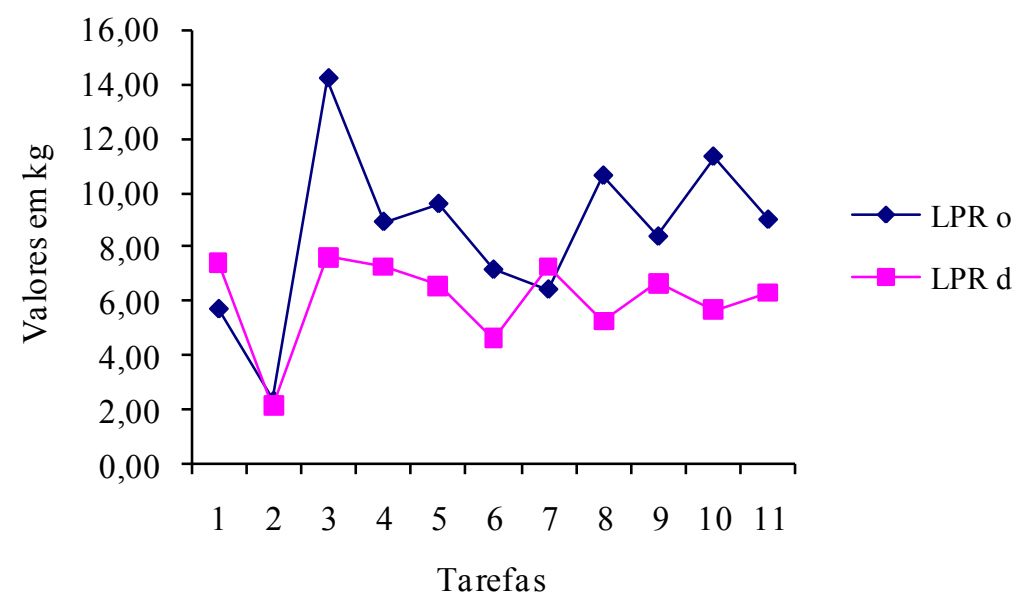

Revista Produção Online. Florianópolis, SC, v.11, n. 3, p. 735-756, jul./set., 2011. 
A afirmação anterior de que as condições das tarefas apresentaram-se mais desfavoráveis no destino dos levantamentos pode ser confirmada mediante os valores do IL, observados no gráfico 5 . Nas oito tarefas - T3, T4, T5, T6, T8, T9, T10, T11- o valor de IL foi mais elevado no destino.

Gráfico 5 - Valores do IL na origem e destino do levantamento

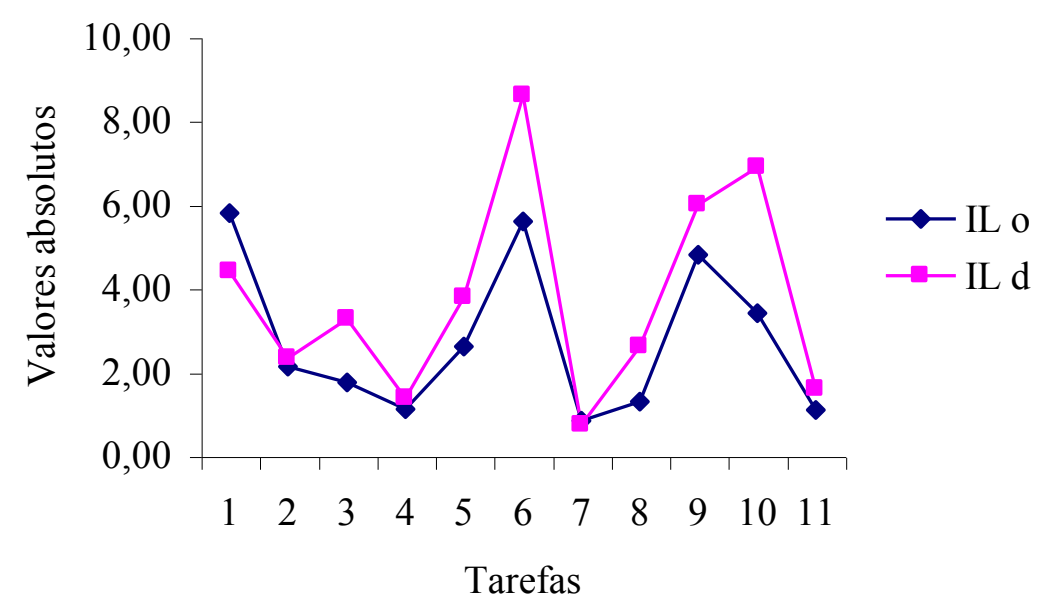

Na tabela 2, observam-se que 17 trabalhadores que realizavam tarefas com ILC no intervalo $1<$ ILC $\leq 3,15(31,25 \%)$ não apresentaram lombalgia relacionada ao trabalh , e dos que 31 trabalhadores que realizavam tarefas com ILC no intervalo $1<\mathrm{ILC} \leq 3$, 28 (58,33\%) não apresentaram lombalgia relacionada ao trabalho.

Tabela 2 - Frequência de lombalgia relacionada ao trabalho nos últimos doze meses por ilc

\begin{tabular}{lccc}
\hline \multicolumn{1}{c}{ ILC } & $\begin{array}{l}\text { Lombalgia relacionada ao trabalho } \\
(-)\end{array}$ & $\begin{array}{l}\text { Lombalgia relacionada ao trabalho } \\
(+)\end{array}$ & $\begin{array}{l}\text { Total da } \\
\text { linha }\end{array}$ \\
\hline $1<$ ILC $\leq 3$ & 15 & 2 & 17 \\
& $31,25 \%$ & $4,17 \%$ & $35,42 \%$ \\
ILC $>3$ & 28 & 3 & $64,58 \%$ \\
& $58,33 \%$ & $6,25 \%$ & 48 \\
Total da & 43 & 5 & $100,00 \%$ \\
\hline coluna & $89,58 \%$ & $10,42 \%$ & \\
\hline
\end{tabular}


Após análise estatística dos dados da tabela 2, concluiu-se que os valores de ILC das tarefas não apresentaram relação significativa com a incidência de lombalgia relacionada ao trabalho nos últimos doze meses, pois o Teste do Quiquadrado apresentou valor de 0,05 com um valor de $p=0,82$, e o Teste $R$ de Pearson foi de $-0,03$ com $p=0,41$.

Na tabela 3, observa-se a frequência de lombalgia por acidente de trabalho nos últimos doze meses de acordo com o ILC. Dos 48 trabalhadores, 17 realizavam tarefas com ILC no intervalo $1<$ ILC $\leq 3$, e apenas um $(2,08 \%)$ dos 17 apresentou lombalgia. Trinta e um dos 48 trabalhadores realizavam tarefas com ILC> 3 e apenas um (2,08\%) apresentou lombalgia. Não houve diferença entre a incidência de lombalgia por acidente de trabalho nos diferentes grupos de acordo com o ILC.

Tabela 3 - Frequência de lombalgia por acidente de trabalho nos últimos doze meses por ILC

\begin{tabular}{lccc}
\hline ILC & LOMBALGIA POR AT (-) & LOMBALGIA POR AT (+) & TOTAL DA LINHA \\
\hline $1<$ ILC $\leq 3$ & 16 & 1 & 17 \\
& $33,33 \%$ & $2,08 \%$ & $35,42 \%$ \\
ILC $>3$ & 30 & 1 & 31 \\
& $62,50 \%$ & $2,08 \%$ & $64,58 \%$ \\
\hline Total da coluna & 46 & 2 & 48 \\
\hline
\end{tabular}

Os valores de ILC das tarefas avaliadas não apresentaram relação significativa com a incidência de lombalgia por acidente de trabalho nos últimos doze meses, pois o Teste do Qui-quadrado apresentou valor de $0,19 \mathrm{com}$ um valor de $p=$ 0,65 e o Teste R de Pearson foi de $-0,06$ com $p=0,33$.

As tarefas de levantamento nas indústrias pesquisadas, geralmente, eram desempenhadas por trabalhadores jovens, do sexo masculino e com peso adequado para a estatura.

A incidência de lombalgia nos últimos doze meses foi de $19 \%$, enquanto que a incidência de lombalgia relacionada ao trabalho no mesmo período foi de $10 \%$. 
Não existiu correlação estatística significativa entre lombalgia e o ILC na amostra estudada. O turnover mostrou-se elevado, o que pode justificar a inexistência de correlação entre lombalgia e ILC.

As tarefas avaliadas apresentaram-se bastante desfavoráveis, principalmente no destino do levantamento, ocasionando alto risco para os trabalhadores, como foi evidenciado mediante os elevados valores do ILC encontrados. Em $72,7 \%$ das tarefas o ILC foi superior a três, sendo as mesmas classificadas como de alto risco ergonômico.

As variáveis da tarefa que mais contribuíram para os valores inadequados de LPR e IL foram: distância horizontal $(H)$, frequência de levantamentos $(F)$ e a distância vertical (V), além do peso elevado da carga.

A ELN para avaliação das tarefas de levantamento de carga é uma ferramenta que não demanda a utilização de tecnologia sofisticada ou aparelhos de alto custo. Contudo, requer do analista conhecimento aprofundado da sua metodologia e dos critérios envolvidos em sua concepção. Exige, também, treinamento sobre o procedimento de obtenção das variáveis no local de trabalho, assim como familiaridade com o manuseio dos instrumentos necessários.

A equação fornece dados importantes sobre as tarefas avaliadas, pois a magnitude relativa de cada fator indica a contribuição relativa de cada variável da tarefa. Assim, foi possível verificar quais as variáveis que mais contribuíram para os valores desfavoráveis do LPR e do IL. Esses dados podem ser utilizados para a realização de melhorias nas tarefas. O IL pode ser usado para estabelecer prioridades de novos projetos ergonômicos, estabelecendo-se um ranking de acordo com os valores de IL.

Cabe salientar que a os resultados desta pesquisa quanto à relação entre o IL da ELN e a incidência de lombalgia descreveram um fenômeno que ocorreu na amostra, porém não podem ser extrapolados para a população geral de trabalhadores das tarefas de levantamento de cargas. 


\section{CONSIDERAÇÕES FINAIS}

Sugere-se às empresas que possuem tarefas de levantamento manual de cargas incluir no Programa de Prevenção de Riscos Ambientais (PPRA) o mapeamento dessas tarefas de acordo com os Índices de Levantamento (IL). Dessa forma, torna-se possível verificar quais são as tarefas mais desfavoráveis e priorizar as melhorias de acordo com os valores de IL apresentados, além de evidenciar as variáveis mais desfavoráveis de cada tarefa. Por meio desse mapeamento, o Médico do Trabalho terá informações importantes sobre os riscos que os trabalhadores estão expostos. Através do PPRA e do Programa de Controle Médico de Saúde Ocupacional (PCMSO), esses riscos poderão, então, ser prevenidos e controlados.

Salienta-se aos usuários que irão utilizar pela primeira vez a equação, a importância de um estudo aprofundado dos conceitos envolvidos, da metodologia de aplicação e do procedimento de obtenção das variáveis em campo para garantir precisão às medições.

\section{REFERÊNCIAS}

BURDORF, A .; VAN DER BEEK, A . Letter to the Editor: accuracy of measurements for the revised $\mathrm{NIOSH}$ lifting equation. Applied Ergonomics, v.29, n.6, p. 433-438, 1998.

BYRNS, G.E.; BIERMA, T.J.; AGNEW, J.; CURBOW, B. A new direction in low back pain research. AlHA J, v 63, n. 1, p. 55-61, 2002.

CHUNG, M.K.; KEE, DOHYUNG. Evaluation of lifting tasks frequently performed during fire brick manufacturing process using $\mathrm{NIOSH}$ lifting equations. Int. Journal of Industrial Ergonomics, v. 25, p. 42 - 433, 2000.

COUTO,H.A. Biomecânica da coluna vertebral e ergonomia na prevenção de lombalgias.In: 1995. v.1, p.185-255.

DEMPSEY, P.G. Usability of revised NIOSH lifting equation. Ergonomics, v. 45, n. 12, p. 817-828, 2002.

DEMPSEY, P.G.; BURDORF, A. ; FATHALLAH, F.A .; SOROCK, G.S.; HASHEMI, L. Influence of measurement accuracy on the application of the $1991 \mathrm{NIOSH}$ equation.

Applied Ergonomics, v. 32, n. 1, p. 91-99, 2001. 
GARG, A . Revised NIOSH equation for manual lifting: a method for job evaluation. AAOHN J, v. 43, n. 4, p. 211-216, 1995.

HARTVIGSEN, J.; BAKKETEIG, L.S.; LEBOEUF-YDE, C.; ENGBERG, M.; LAURITZEN, T. The association between physical workload and low back pain clouded by the "healthy worker" effect: population-based cross-sectional and 5-year prospective questionnaire study. Spine, v. 26, n. 16, p. 1788-1793, 2001.

KERR, M.S.; FRANK, J.W.; SHANNON, H.S.; NORMAN, R.W.; WELLS, R.P.; NEUMANN, W.P.; BOMBARDIER, C. Biomechanical and psychosocial risk factors for low back pain at work. Am J Public Health, v. 91, n. 7, p. 1069-1075, 2001.

LIN, Y.H.; CHEN, C.S.; CHEN, W.J.; CHENG, C.K. Caracteristics of manual activities in the pacients with low-back pain. International Journal of Industrial Ergonomics, v. 29, p. $101-106,2002$.

MARRAS, W. S. Occupational low back disorder causation and control. Ergonomics, v.43, n.7, p.880-902, 2000.

MARRAS, W.S.; FINE, L.J., FERGUSON, S.A .; WATERS, T.R. The effectiveness of commonly used lifting assessment methods to identify industrial jobs associated with. Applied Ergonomics, v. 30, n. 4, p. 369-370, 1999.

NATIONAL INSTITUTE FOR OCCUPATIONAL SAFETY AND HEALTH (NIOSH). Applications manual for the revised NIOSH lifting equation. U.S. Dept. of Health and Human Services (NIOSH), Public health Service, Cincinnati, OH, 1994.

OKIMOTO, M. L. L.R.; TEIXEIRA, E.R. Proposed procedures for measuring the lifting task variables required by the revised niosh lifting equation: a case study.

International Journal of Industrial Ergonomics v.39, n.1, p. 15-22, 2009.

PI-SUNYER, F. X. Obesidade. In: WYNGAARDEN, J. B.,SMITH, L. H.Cecil tratado de medicina interna. 18. ed. Rio de Janeiro: Guanabara Koogan S.A.,1990.

QUINN, P. R. Returning to work after disability. Employee Benefits Journal, v.27, n.2, p.13-17, 2002.

TEIXEIRA, E.R. Sistematização de procedimentos necessários à aplicação da ELN: estudo descritivo da relação entre o IL da equação revisada do NIOSH e a incidência de lombalgia numa amostra de trabalhadores. 2004. $224 \mathrm{f}$.

Dissertação (Mestrado em Engenharia Mecânica) - Setor de Tecnologia, Universidade Federal do Paraná, Curitiba.

THORBJORNSSON, C.O.B.; ALFREDSSON, L.; FREDRIKSSON, K.; MICHELSEN, H.; PUNNETT, L.; VINGARD, E.; TORGÉN, M.; KILBOM, A . Physical and psychosocial factors related to low back pain during a 24-year period. A nested casecontrol analysis. Spine, v. 25, n. 3, p. 369-375, 2000. 
TULDER, M. V., KOES, B., BOMBARDIER, C. Low back pain. Best Practice \& Research Clinical Rheumatology, v.16, n.5, p.761-775, 2002.

WATERS, T. R., PUTZ-ANDERSON, V., GARG, A., FINE, L. J. Revised NIOSH equation for design and evaluation of manual lifting tasks. Ergonomics, v.36, n.7, p.749-776, 1993.

WATERS, T. R.; PUTZ-ANDERSON, V.; BARON, S. Methods for assessing the physical demands of manual lifting: A review and case study from warehousing. Am. Ind. Hyg. Assoc. J., v.59, n.12, p.871-881, 1998.

WATERS, T.R., Reply to the letter by Drs Burdorf and Van Der Beek, Applied Ergonomics, 1999, 30,369-370. Applied Ergonomics, v.30, n. 4, p.371, 1999b.

WATERS, T.R.; BARON, S.L.; KEMMLERT, K. Accuracy of measurements for the revised NIOSH lifting equation. National Institute for Occupational Safety and Health. Applied Ergonomics, v. 29, n. 6, p. 433-438, 1998.

WATERS, T.R.; BARON, S.L.; PIACITELLI, L.A .; ANDERSON, V.P.; SKOV, T.; HARING-SWEENEY, M.; WALL, D.K.; FINE, L.J. Evaluation of the revised NIOSH lifting equation. A cross-sectional epidemiologic study. Spine, v. 24, n. 4, p. 386-394, 1999a.

YEUNG, S. S.; GENAID, A . M.; KARWOWSKI, W.; LEUNG, P. C. Reliability and validity of self - reported assesssment of exposure and outcome variables for manual lifting tasks: a preliminary investigation. Applied Ergonomics, v.33, n. 5, p.463 469, 2002.

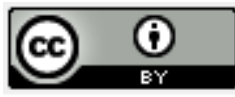

Artigo recebido em 21/06/2010 e aceito para publicação em 17/08/2011. 